\title{
Proposta de um construto para gestão da informação no ciclo de vida dos agentes
}

\section{Sergio Evangelista Silva ${ }^{I}$}

http://orcid.org/0000-0002-3507-518X

Filipe Nunes Ribeiro ${ }^{I I}$

Joao Alexandre Borges Garcia ${ }^{I I I}$

Vicente José Peixoto de Amorim ${ }^{I V}$

Rafael Ferreira Camardav

${ }^{I}$ Universidade Federal de Ouro Preto, Ouro Preto, MG, Brasil.

Docente do Departamento de Engenharia de Produção da Universidade Federal de Ouro Preto.

${ }^{I I}$ Universidade Federal de Ouro Preto, Ouro Preto, MG, Brasil. Doutorando em Ciência da Computação pela Universidade Federal de Minas Gerais.

III Universidade Federal de Ouro Preto, Ouro Preto, MG, Brasil. Graduado em Engenharia de Produção pela Universidade Federal de Ouro Preto.

IV Universidade Federal de Ouro Preto, Ouro Preto, MG, Brasil. Doutorando em Ciência da Computação pela Universidade Federal de São Carlos.

$\checkmark$ Universidade Federal de Ouro Preto, Ouro Preto, MG, Brasil. Graduado em Engenharia da Computação pela Universidade Federal de Ouro Preto.

http://dx.doi.org/10.1590/1981-5344/2796

Atualmente as infromações geradas por um agente (ex. uma pessoa; um automóvel; uma obra pública, etc.) ao longo do seu ciclo de vida encontram-se espalhadas por diferentes sistemas de informação (SIs) de diferentes organizações. Por exemplo, um cidadão ao longo de sua vida gera informações nos mais variados tipos de organização (ex. hospitais, escolas, empresas, repartições públicas, etc.). Todavia, estas informações contidas nos 
SIs destas organizações não podem ser livremente acessadas por este cidadão. Contudo, frente ao atual desenvolvimento tecnológico é possível desenvolver SIs capazes de centralizar em um único respositório as informações geradas por um agente ao longo do seu ciclo de vida, construção essa ainda pouco observada em nossa sociedade. Em face desta oportunidade de pesquisa neste artigo é proposto um construto para gestão da informação no ciclo de vida dos agentes (GICVA). Tendo em vista seu caráter aplicado é utilizado o método Design Science Research (DSR). Em seguida, este construto é demonstrado por meio de um SI para gestão de informações de pessoas em dois contextos. Da perspectiva conceitual este artigo apresenta um novo construto que permitirá desenvolver SIs sob um novo paradigma. Da perspectiva da aplicação, SIs baseados nos princípios da GICVA poderão ser implementados para gerenciar informações de diferentes tipos de agentes, tais como pessoas, automóveis, imóveis, projetos, dentre outros, podendo trazer grandes benefícios para a sociedade.

Palavras-chave: gestão da informação; gestão de documentos; sistemas de informação.

\section{Proposal of a construct for information management in the agent life-cycle}

Currently, information generated by an agent (e.g., a person; a vehicle; a public project, etc.) across its life cycle is scattered in different information systems (ISs) of different organizations. For instance, a citizen during his life generates information in various kinds of organizations (e.g., hospitals, schools, firms, public bureaus, etc.). However, the information inserted in the ISs of these organizations cannot be freely accessed by this citizen. Nevertheless, referring to the current technological development, it is possible to develop ISs capable of centralizing in a unique repository the information of different kinds of agents, across their lifecycle. Owing to this opportunity of research in this 
article is introduced a construct for information management in the agent lifecycle (IMALC). Considering its applied character, it is used the Design Science Research (DSR) method. This construct is also demonstrated through an application for information management of persons, in two contexts of day-to-day life. From the conceptual perspective, this article presents a new construct for the development of ISs, under a new information management paradigm. From the applied research viewpoint, ISs which incorporate the IMALC principles can be applied to the management of information of different types of agents, such as, persons, automobiles, buildings, projects, and so on, bringing great benefits for the society.

Key-words: information management; document management; information systems.

Recebido em 22.04.2019 Aceito em 16.05.2019

\section{Introdução}

Atualmente as informações documentais e não documentais relativas às pessoas, organizações e objetos em geral encontram-se distribuídas em diversos sistemas de informação (SIs) de diferentes organizações (LAUMER et al., 2013). Toda informação diz respeito ao registro, por meio do uso da linguagem escrita, de sons, ou de imagens de um determinado fenômeno, ou estado relativo a um objeto. A informação pode ser documental, ou seja, quando devido à sua relevância, deve ser gerada uma certidão que comprova a ocorrência de um determinado evento (uma nota fiscal de compra; o comprovante de depósito bancário; etc.), ou não documental, que consiste no registro para o qual não é gerada certidão, tendo em vista que a priori, não se necessita comprovação da ocorrência. Para fins de simplificação do texto que se segue, doravante o termo informação se referirá a estes dois subtipos apresentados.

$\mathrm{Na}$ atualidade o paradigma de SI vigente está centrado em uma visão da informação como propriedade restrita à organização (LAW; NGAI, 2007; BOBSIN et al. 2010; MORESI et al., 2010; ESPANTOSO, 2012; JANUZZI et al. 2014). Por exemplo, um cidadão gera informações nos mais variados contextos de sua vida (ex. saúde, profissional, estudantil, etc.), que encontram-se dispersas em SIs de diversas organizações, tais como: bancos, escolas, governos, hospitais, empresas, dentre outras. Com efeito, dado o atual estado de desenvolvimento dos SIs este cidadão não possui livre acesso a estas informações no cenário atual, a não ser que elas estejam em sua posse na forma de documentos impressos em papel. 
O considerável nível de desenvolvimento das várias tecnologias de informação e comunicação (TICs), especificamente a computação ubíqua (MAASS; VARSHNEY, 2012) e a computação na nuvem (ARMBRUST et al., 2010; CHANG et al., 2013), já possibilita a infraestrutura para o desenvolvimento de tecnologias mais eficazes para a gestão das informações do cidadão, e outros tipos de agentes geradores de informação (AGIs) (ex. pessoas; imóveis; organizações, veículos, projetos, dentre outros). Como os exemplos sugerem, um AGI consiste em uma entidade, cuja a gestão da informação ao longo de todo o ciclo de vida é relevante, seja para pessoas, para o Estado, ou outro tipo de organização.

Não obstante, as possibilidades tecnológicas oferecidas pelas atuais TICs apresentam novos desafios e possibilidades para a ciência da informação, abrindo possibilidade para o desenvolvimento de novas formas de pensar, ou seja, novos paradigmas para a gestão da informação.

Apesar de todo o desenvolvimento apresentado, não se observa nenhuma tecnologia que atualmente permita, a partir de um único ambiente e ponto de referência, a gestão de todas as informações de um AGI, ao longo do seu ciclo de vida. Todavia, iniciativas como a geração por governos de um número de registro único do cidadão apontam para o desenvolvimento de tecnologias que caminharão neste setido. Por exemplo, govermos de países como India (BHATIA; BHABHA, 2017; CHAUHAN; KAUSHIK, 2016) e Dinamarca (THYGESEN et al., 2011) têm realizado esforços no sentido de se desenvolver SIs centralizados, capazes de armazenar as informações do cidadão. Embora tais progressos sejam notáveis, algumas lacunas ainda permanecem, a saber: o desenvolvimento de tais sistemas se mostra direcionado ao fortalecimento do controle do Estado sobre o cidadão, e não no sentido de permitir que o cidadão possa ter livre acesso às informações que Ihe dizem respeito; esta é uma visão centrada na gestão das informações geradas pelo cidadão no seu contato com órgãos da administração pública direta, ou indireta (ex. agentes de saúde), não estando voltada para a gestão em outros contextos da sua vida, como, por exemplo, na sua relação com empresas, e outros tipos de organizações não governamentais; esta é uma visão centrada na pessoa, não havendo uma abordagem conceitual geral que permita gerar SIs capazes de gerenciar a informação no ciclo de vida de outros tipos de AGIs, tais como, organizações, objetos móveis (veículos, aparelhos eletrônicos, obras de arte, equipamentos, etc.), imóveis e construções (casas, prédios, obras públicas).

Em face das lacunas apresentadas acima, e do estado atual de desenvolvimento das TICs, pode-se verificar a oportunidade de desenvolvimento de novos SIs que permitam o gerenciamento da informação de AGIs, ao longo de todo o seu ciclo de vida. Esta abordagem exige o desenvolvimento de SIs que transcendam os limites de uma organização, o que já é possível graças à internet, e a tecnologias como a computação na nuvem, e a computação ubíqua. Todavia, é necessário 
para isso o desenvolvimento de novos construtos que permitam uma nova forma de pensar sobre a gestão da informação. Frente a estas considerações este artigo propõe um novo construto baseado no conceito de gestão da informação no ciclo de vida dos agentes (GICVA). Ele consiste em um novo paradigma para o desenvolvimento de SIs capazes de gerenciar as informações de diferentes tipos de AGIs, ao longo do seu ciclo de vida (criação/nascimento; crescimento/desenvolvimento; morte/descarte; pós-morte/pós-descarte). Como abordagem metodológica foi utilizado o método Design Research Science (DSR) que na visão de diversos autores é o mais adequado à proposição de artefatos inovadores, dedicados à resolução de um problema contemporânero relevante, que também possui grande demanda social (HEVNER et al., 2004; HOPPEN et al., 2017; LACERDA et al., 2013; SORDI et al., 2015).

Este construto tem como função orientar analistas e desenvolvedores de SIs para o desenvolvimento de sistemas que permitam a gestão da informação de algum tipo de AGI. Por seu turno, podem ser implementados SIs sob os princípios da GICVA que poderão ser utilizados pelas pessoas, nos seus variados papéis sociais (cidadão, funcionário de uma empresa, médico, paciente, etc.) e pelas organizações para gerenciar informações que de alguma forma Ihes dizem respeito.

Doravante este artigo encontra-se organizado da seguinte forma. É inicialmente apresentada uma revisão da literatura sobre gestão da informação e SIs. Em seguida, na terceira seção é apresentado o método utilizado na pesquisa. Na quarta seção é apresentado o construto com os principais elementos da GICVA. Na quinta seção o construto é demonstrado por meio da apresentação de um SI, que é capaz de gerenciar informações de pessoas em dois diferentes contextos. Na sexta seção são analisados os resultados da pesquisa. A sétima seção encerra o artigo com conclusões e sugestões para pesquisas futuras sobre a GICVA.

\section{Referencial teórico}

\subsection{Informação documental e não-documental}

Dentre as visões sobre o conceito de informação há que se distinguir duas vertentes, aquela que se concentra em definir a informação a partir de uma perspectiva ontológica. Ou seja, baseada na definição e no estudo do que é a essência da informação. A segunda vertente considera a informação de uma perspectiva instrumental, ou seja, como este elemento é utilizado, e qual o seu papel no desenvolvimento da sociedade.

A partir de uma perspectiva ontológica a informação pode ser concebida como um conjunto de dados capaz de apresentar um determinado significado sobre algum fenômeno (DAVENPORT, 2000; OLIVEIRA, 2001; MAASS; VARSHNEY, 2012). Com efeito, as informações emergem dos fatos, ou seja, dos diversos fenômenos e estados dos elementos da realidade. 
A partir da perspectiva instrumental há que se considerar que desde - surgimento da história diversas civilizações viram a necessidade do registro das informações, situação que perdura até os dias atuais, e que é fundamental no mundo contemporâneo. Não obstante, pode-se considerar dois tipos de informação registrados atualmente, quais sejam, a informação documental, que é aquela emitida por meio de certidões, tanto por órgãos do Estado, quanto por pessoas e organizações. A contínua gênese de informações documentais ocorre por questões legais, ou seja, como forma de comprovar a ocorrência de determinados fatos (RIOS; CORDEIRO, 2010; PRADOS-SUÁREZ et al., 2012; RIBEIRO, 2012), são diversos os tipos de documentos existentes, tais como: diplomas, documentos previdenciários; recibos de pagamento, dentre outros. Em sua maior parte, atualmente estes documentos só possuem validade se emitidos em papel. Contudo, atualmente já se percebe esforços no sentido da contínua digitalização de documentos (ex: ESPANTOSO, 2012; FERRO et al., 2013; FLORES; SANTOS, 2015). Todavia, um desafio neste caso é garantir a autenticidade dos mesmos, bem como um processo seguro para sua gestão e conservação (FLORES; SANTOS, 2015).

No que diz respeito ao segundo tipo de informação, embora haja atualmente a capacidade de governos e outras organizações de gerarem grandes volumes de informação documental, há que se considerar que a realidade é por demasiada extensa para que se possa registrar, e emitir documentos para todas as ocorrências de fatos. Neste sentido, diversas informações geradas nos dias atuais, prescinden da criação de um documento para comprová-las. Por exemplo, cameras de monitoramento de ambientes geram a todo o momento grandes volumes de informação, que não são documentais. Todavia, a necessidade de transformação da informação não-documental em documental (reconhecida por algum agente público, ou privado) dependerá de circunstâncias. Por exemplo, informações contidas em câmeras de monitoramento podem ser transformadas em informações documentais, para a elucidação de um acidente de trânsito.

Tendo em vista a crescente disponibiblidade de diversos tipos de TICs, há que se considerar que os meios digitais serão cada vez mais utilizados para a geração e armazenamento de informações documentais e não-documentais. Todavia, em face do grande volume de informações gerado atualmente, é fundamental o desenvolvimento de artefatos que tornem mais eficiente o processo de gestão de informações. Neste contexto, a GICVA pode ser expressa por meio de um novo construto. Este construto deve apresentar os elementos fundamentais para a implementação de SIs capazes de gerenciar informações de AGIs ao longo de seu ciclo de vida.

\subsection{Paradigmas de gestão e modelagem da informação}

A ideia de paradigma permeia diversos campos da atividade humana, sendo uma construção metafísica formada por um conjunto de 
construtos relacionados a um dado contexto (KISHORE et al., 2006; ASHURST et al., 2012). Em suma, um paradigma se manifesta em um conjunto de premissas utilizadas para se conceber determinado contexto da realidade.

$\mathrm{Na}$ área de gestão da informação os paradigmas estão, sobretudo, associados a modelos de dados, em que se destacam o Modelo EntidadeRelacionamento (CHEN, 1976), que concebe a organização de dados de um determinado contexto, a partir dos seguintes elementos: o conceito de entidade, em que se identificam os entes do contexto (pessoa; produto; etc.); os atributos, que caracterizam uma dada entidade; as relações entre as entidades. Por exemplo, Sales; Sayão (2015) apontam o uso deste modelo em SIs destinados à organização de informações de pesquisas científicas.

Outro paradigma utilizado no desenvolvimento de SIs é o Orientado a Objetos, cujo elemento fundamental consiste nos objetos, que representam elementos da realidade. Eles possuem atributos que os qualificam e realizam ações que também devem ser registradas (KISHORE et al., 2006; SILBERSCHATZ et al., 2006). Nota-se que estes dois modelos consistem em duas formas diferentes de abstração da realidade. Ainda a este respeito Maass, Varshney (2012) propõem a modelagem da informação baseada em duas camadas, a saber, a dos fenômenos da realidade objetiva e a das representações destes fenômenos.

\subsection{Os SIs atuais e suas tendências}

Os SIs se colocam como um instrumento fundamental no processo de gestão da informação, permitindo sua gênese, manipulação, armazenamento e recuperação (CARDOSO; PEREIRA, 2005). Especificamente os SIs são compostos de estruturas de hardware e software de processamento, armazenamento e de comunicação, que permitem o referido processo de gestão da informação (OLIVEIRA, 2001). Destaca-se que majoritariamente a literatura sobre SIs desenvolveu-se em torno de sistemas dedicados ao suporte às organizações (LAW; NGAI. 2007; BOBSIN et al. 2010; ESPANTOSO, 2012; JANUZZI et al. 2014), que é o local em que eles tiveram sua origem.

Dentre os vários SIs dedicados às organizações vale destacar os sistemas ERP (enterprise resource planning), que são dedicados a dar suporte às organizações empresariais, consistindo na maior expressão de sua integração (LAW et al. 2007). Estes sistemas têm como propósito suportar a realização de transações nas diversas funções e níveis hierárquicos da empresa (BOBSIN et al. 2010).

Tigre, Noronha (2013) apresentam uma clara síntese da evolução dos SIs ao longo do tempo, cujo primeiro estágio consistiu no surgimento de múltiplos SIs independentes (conhecidos como sistemas legados), em que cada SI dava suporte a gestão das informações de uma determinada função empresarial (produção; marketing; pesquisa e desenvolvimento; recursos humanos; finanças e contabilidade), até culminar no 
desenvolvimento dos sistemas ERP, que consistem em sistemas que reúnem as informações de toda a empresa sob um único ambiente de software. Todavia, na atualidade verifica-se a tendência do desenvolvimento de SIs, cujas informações não mais se encontram no hardware das organizações, mas sim em sistemas baseados na computação na nuvem (ARMBRUST et al., 2010; CHANG et al. 2013; TIGRE \& NORONHA, 2013), ou seja, em espaços de dados remotos, externos à organização.

Outro aspecto dos atuais SIs é que eles permitem a troca de informações entre organizações (KISHORE et al., 2006; JANUZZI et al., 2014). Outra tendência é o desenvolvimento de SIs e modelos de dados baseados em contextos, ou seja, determinados domínios semânticos, cujo propósito é facilitar o armazenamento e recuperação da informação (ex. PRADOS-SUÁREZ et al. 2012; NADOVEZA; KIRITSIS, 2014; GULDENPFENNING; FITZPATRICK, 2015).

Quanto ao conceito de AGI apresentado na introdução deste artigo, ainda são recentes e escassos os esforços cujo foco central é associar sempre ao AGI às informações que ele gerou (ex. BRENMAN; CASPER, 2015; DOBBINS et al., 2014; GULDENPFENNING; FITZPATRICK, 2015; SALES; SAYÃO, 2015). No Brasil vale destacar o trabalho de Sales; Sayão (2015) na área de documentação e informação científica, em que eles apontam para o desenvolvimento de aplicações integradas em diversos níveis de escopo, que permitam gerenciar a informação, nas palavras destes autores a partir do "ciclo de vida das atividades de pesquisa".

Também se tem observado o esforço de alguns países na criação de um identificador único para seus cidadãos. Por meio do esquema Aadhar a India tem promovido um esforço de criação de um identificador único para gerenciar informações de seus cidadãos, a partir de uma base de dados única (BHATIA; BHABHA, 2017; CHAUHAN; KAUSHIK, 2016). Nesta mesma linha de ação a Dinamarca tem direcionado esforços na criação de um identificador único do cidadão para gerenciar principalmente registros de saúde e sociais (THYGESEN et al., 2011). Estes últimos trabalhos sinalizam que um paradigma como a GICVA possuem grande potencial, no sentido de direcionarem o desenvolvimento de SIs capazes gerenciar as informações do cidadão e outros AGIs, para além dos limites de uma única organização.

\section{Método}

Tendo em vista que o objetivo deste artigo é propor um construto para o desenvolvimento de SIs sob os princípios da GICVA, o método que melhor dá base para esta pesquisa é a Design Science Research (DSR) (HEVNER et al., 2004; GREGOR; HEVNER, 2013; SORDI; AZEVEDO, 2013; SORDI et al., 2013), que é uma modalidade de pesquisa adequada a área de SIs, uma vez que seu propósito fundamental é dar embasamento científico ao desenvolvimento de novas soluções tecnológicas, que se disponham a solucionar problemas de grande relevância para a sociedade. 
Com efeito, esta pesquisa possui um caráter de acão humana na realidade, qual seja, a de desenvolvimento de um construto, neste caso, um elemento que não é natural, com o propósito de resolver determinado problema de alta relevância para a sociedade, visão esta em consonãncia com diversos autores da DSR (ex. LACERDA et al., 2013; GREGOR; HEVNER, 2013; SORDI et al., 2015).

O propósito da DSR é a gênese de artefatos destinados à resolução de determinado problema. Neste sentido, são quatro os tipos de artefatos de SIs a serem produzidos no âmbito da DSR (HEVNER et al., 2004; LACERDA et al., 2013): os construtos que consistem em um conjunto de termos que formam um vocabulário em determinado domínio; os modelos, que consistem em abstrações e representações da realidade que expressam as relações entre construtos; os métodos, que são algoritmos e práticas para se atingir um determinado objetivo; e as instanciações, que consistem na "materialização" dos três tipos anteriores de artefatos em um determinado domínio de aplicação.

Para a aplicação do método DSR Peffers et al., (2007) propõem as seguintes etapas: 1 - Identificação do problema e motivação; 2 Definição dos objetivos para a solução; 3 - Projeto e desenvolvimento, que identifica os artefatos a serem desenvolvidos (construto; modelo; método ou instância); 4 - Demonstração, aplicação do artefato desenvolvido em alguma situação real; 5 - Avaliação, observação e medição rigorosa do artefato na solução do problema; 6 - Comunicação, que consiste na redação de um documento informando a comunidade científica acerca do artefato desenvolvido. À luz da tipologia de Peffers et al., (2007) são apresentados respectivamente os seguintes processos desenvolvidos nesta pesquisa: o problema que motivou esta pesquisa consiste no fato que atualmente as informações de diferentes tipos de AGIs encontram-se espalhadas em SIs de diferentes organizações, gerando diversos problemas e custos para sua recuperação. Com efeito, o desenvolvimento de SIs capazes de centralizarem estas informações em repositórios de informações seria de grande valia para a sociedade; 0 objetivo deste trabalho é propor um construto para o desenvolvimento de SIs segundo a perspectiva da GICVA; além do projeto do construto neste artigo também é apresentada a demonstração de seu uso, por meio de um SI laboratorial para a gestão das informações de pessoas em dois contextos, a saber: o contexto de contratos composto por um subcontexto, o de locação de imóveis; e o contexto de saúde, formado pelos subcontextos exames e monitoramento de dados biológicos; tendo em vista o objetivo e a extensão deste artigo optou-se por não se fazer uma análise rigorosa do construto, e nem do SI implementado. Dada a dimensão destas atividades, elas deverão motivar uma nova pesquisa com este fim específico no futuro; este documento consiste em um dos primeiros meios de comunicação do construto, e dos conceitos relativos à GICVA, devendo servir de referência para publicações futuras neste assunto. 


\section{A Apresentação do Construto sobre a GICVA}

A GICVA é baseada em três camadas: 1 - os elementos da realidade de interesse; 2 - os elementos do ambiente informacional; 3 - e os elementos do ambiente tecnológico.

\subsection{Elementos da realidade de interesse}

É importante distinguir claramente entre o fenômeno que ocorre na realidade concreta, ou seja, no mundo físico e a sua representação enquanto informação, como faz o modelo de Maass; Varshney (2012). Neste sentido, entende-se por realidade de interesse um domínio a ser atendido por um SI sob a perspectiva da GICVA. Esta realidade compreende tanto elementos tangíveis, concretos, tais como automóveis, imóveis, pessoas, quanto elementos intangíveis, abtratos, tais como normas, textos, etc.

No que tange a GICVA os construtos relativos à modelagem da realidade objetiva são:

Entidade: Elemento da realidade de interesse (ex. automóvel; produto de consumo; pessoa, etc.).

Agente Gerador de Informação (AGI): Subconjunto da classe de todas as entidades. O AGI consiste em um elemento de alta relevância, sobre o qual é importante gerenciar informações. Além do cidadão, são exemplos de AGIs, as organizações, os bens de alto valor e/ou relevância social, por exemplo, veículos, equipamentos, imóveis, animais, grandes obras, dentre outros.

Evento: Consiste na ocorrência de um fenômeno em um determinado momento, ao qual está obrigatoriamente associado pelo menos um AGI, podendo envolver também objetos. São exemplos, a compra de um produto por uma pessoa, a matrícula em uma escola, a quebra de um equipamento, dentre outros.

Eventos fundamentais: São relativos ao surgimento, ou à extinção de um AGI. Considerando uma determinada pessoa, o evento de surgimento é o seu nascimento, e a extinção a sua morte, encerrando o seu ciclo de vida.

Eventos contextuais: Após o evento de surgimento, o AGI realizará e/ou sofrerá eventos em diversos contextos do seu ciclo de vida. Por exemplo, considerando-se uma determinada pessoa, ao longo de sua vida ela noralmente passará por consultas médicas, estudará, trabalhará, dentre outras atividades. 
Com efeito, os eventos contextuais podem ensejar a gênese de informações que devem ser armazenados em um único repositório, garantindo o seu resgate no futuro. A classificação dos contextos em que os eventos ocorrem é importante para que se possa armazenar e recuperar a informação, com maior facilidade.

\subsection{Elementos do ambiente informacional}

Os principais construtos relativos ao ambiente informacional são:

Abstração de dados: A informação representa apenas um aspecto do AGI a qual ela se refere, e não o seu todo. Edmund Hurssel (HURSSEL, 2006) precursor da fenomenologia deixa claro que são inúmeras as formas de percepção de determinado objeto da realidade. Conclui-se, a partir deste princípio, que são inúmeras as formas de representação de um determinado objeto da realidade, por meio da informação. Com efeito, todo processo de modelagem da informação deve ser norteado por um processo de abstração (SILBERSCHATZ et al., 2006), em que se define quais os elementos de informação do AGI serão armazenados.

Atributo ou elemento de dado: Consiste em um elemento que caracteriza o AGI (SILBERSCHATZ et al. 2006). Por exemplo, os atributos de caracterização de um imóvel são: o endereço; a natureza do mesmo; a área; a localização; dentre outros.

Registro de evento fundamental: Este registro está associado ao surgimento, ou à extinção de determinado AGI. Ele será formado pela composição de um conjunto de elementos de dados, que caracterizam aspectos básicos da origem, ou extinção do AGI. Para o caso da pessoa, o registro de evento fundamental de criação deveria conter os dados da certidão de nascimento, enquanto o de extinção, os dados da certidão de óbito.

Registro de evento contextual: O conceito de GICVA pressupõe o armazenamento em um único ambiente de dados, de todas as informações relevantes, relativas a um determinado AGI. Estas informações serão geradas nos mais variados contextos do seu ciclo de vida. A pessoa, por exemplo, gera informações ao longo de sua vida, relativas a contextos como o cível (certidões e documentos pessoais); o estudantil (diplomas, históricos e documentos estudantis em vários níveis); financeiro (recebimentos, compras, contratos,recibos,...); 0 profissional (contratos de trabalho; holerites; atestados; e outros documentos trabalhistas); e de saúde (consultas; receitas médicas; internações), dentre outros. Já um 
automóvel normalmente gerará informações nos contextos legal (registro do veículo; seguro obrigatório; pagamentos de impostos); de transações econômicas (mudança de posse); e de manutentção (registros de manutenção; registro de peças trocadas, etc.).

\subsection{Elementos tecnológicos}

O terceiro nível constitutivo da GICVA é formado pelos elementos tecnológicos. São estes elementos que constituem a estrutura de dispositivos e equipamentos, que permitem a captação de dados no ambiente real, e o seu devido registro no ambiente de computação na nuvem. São apresentados a seguir os principais elementos:

Hardware: É formado pelos dispositivos físicos, tais como, computadores, dispositivos móveis e sensores, que permitem todo o processo de gestão da informação e de documentos;

Dispositivos móveis: Dentro do contexto do hardware merecem destaque os dispositivos móveis. São exemplo destes dispositivos aparelhos de telefonia celular e tablets, sendo fundamentais em um cenário em que se deseja registrar todas as informações relevantes de um determinado AGI. Isso porque são objetos portáteis, podendo sempre estar próximos ao AGI. Há que se citar também o surgimento de dispositivos para o registro de eventos, que consistem em sensores e estruturas para o acoplamento dos mesmos, tais como roupas, ou estruturas para serem vestidas pelo indivíduo (wereable) (PRADOS-SUÁREZ et al., 2012; DOBBINS et al., 2014), capazes de captar diversos tipos de informação em tempo real.

Software: Consiste nos conjuntos de instruções que operam os dispositivos de hardware, realizando o processo de gestão da informação;

Computação na nuvem: O registro de todas as informações relevantes de um AGI necessitará de um ambiente único, em que se possa fazer apenas um registro relativo ao mesmo. Atualmente são os ambientes de computação na nuvem, presentes na internet os elementos capazes de dar suporte a este objetivo (ARMBRUST et al., 2010; CHANG et al. 2013; TIGRE \& NORONHA, 2013).

A Figura 1 apresenta um esquema conceitual do construto acima proposto, no sentido de facilitar a compreensão e as relações entre os seus elementos. Tem-se neste esquema três grandes blocos formados pelos elementos da realidade de interesse, que constituem os elementos 
da realidade objetiva na qual determinado AGI está inserido. É neste ambiente que os eventos são gerados. O ciclo de vida de um determinado AGI tem seu início na ocorrência de um evento fundamental de surgimento, e o seu fim em um evento fundamental de extinção. Ao longo do seu ciclo de vida diversos eventos contextuais são gerados. Estes eventos possuem impacto direto no ambiente informacional, gerando respectivamente o registro de surgimento, diversos registros contextuais, e o registro de extinção do AGI. Todavia, apenas parte da realidade destes eventos é registrada em registros de informação. Este aspecto consiste no processo de abstração, que é simbolizado por uma seta do bloco dos elementos da realidade de interesse para o bloco dos elementos do ambiente informacional.

Todo o processo de gênese e registro de informações na GICVA deve ocorer baseado em um bloco de elementos tecnológicos, formado pelos elementos de hardware de computadores, de comunicação, e dos dispositivos móveis. Dentro destes dispositivos é que funcionam diversos pacotes de software. Neste contexto, todas as informações devem estar armazenadas em ambientes de computação na nuvem, ou seja, em ambientes de informação remotos.

Figura 1 - Estrutura conceitual do construto sobre 


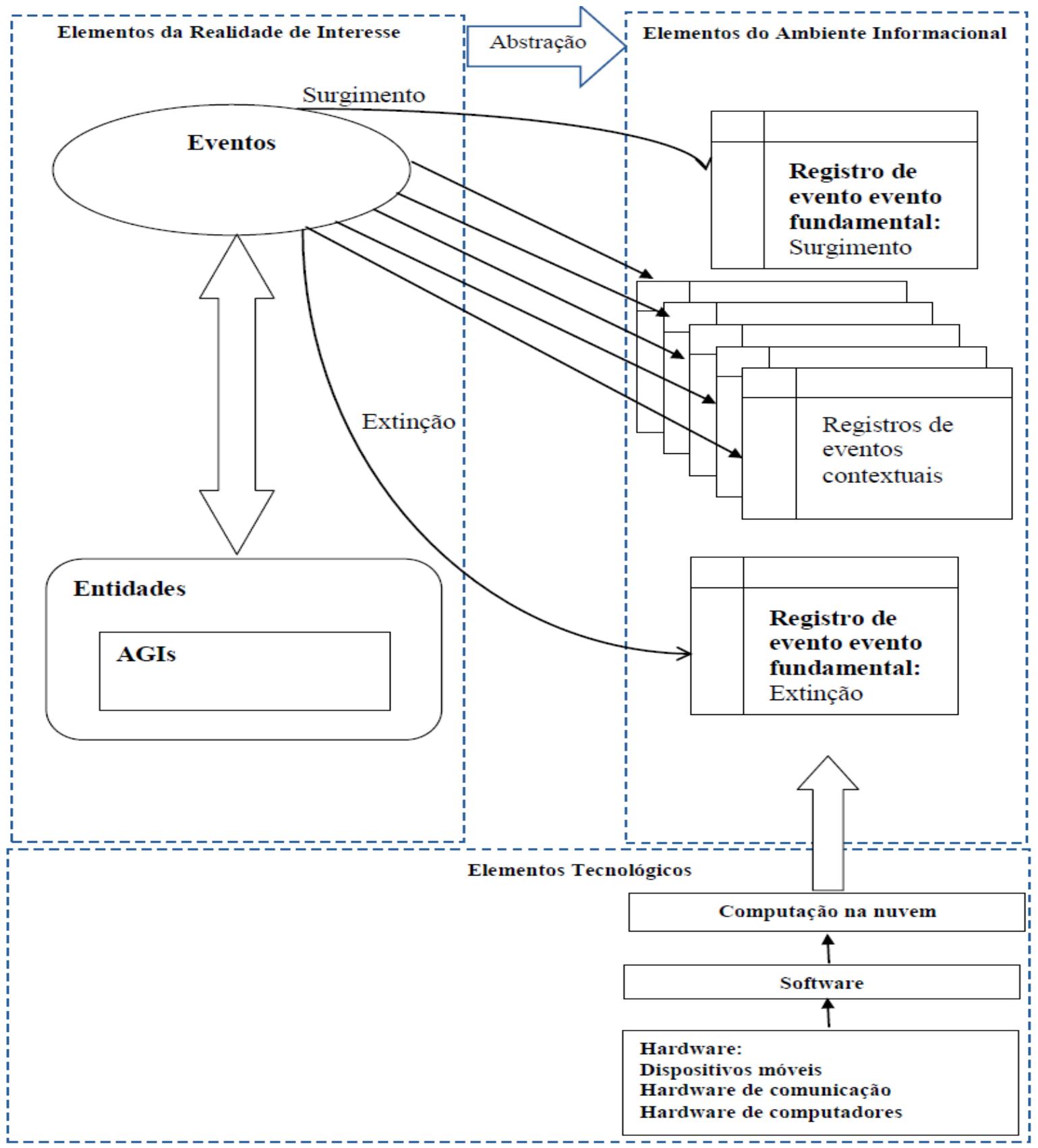

Fonte: os autores

\section{Demonstração da Aplicação do Construto sobre a GICVA}

O processo de validação de teorias e aplicações é bastante comum na área de gestão da informação (DING et al. 2014; MAASS \& VARSHNEY, 2012), sendo algo típico no método DSR (GREGOR, HEVNER, 2013), tendo em vista o seu caráter pragmático. O SI desenvolvido para demonstrar a GICVA consiste em um sistema de armazenamento de informações, baseado em dois contextos, quais sejam, o econômico-financeiro e o de saúde. Este SI será denominado de AmbienteCicloVida. Ele opera em um servidor de internet, apenas em caráter laboratorial, em um ambiente de computação na nuvem. 


\subsection{Modelagem dos elementos da realidade de interesse}

Os principais elementos modelados na implementação do AmbienteCicloVida são apresentados no Quadro 1. Foram modelados três eventos, a realização de contrato de aluguel de imóvel, de exame médico (exame de sangue), e o monitoramento do índice de glicemia de um indivíduo. Neste caso o indivíduo é responsável por monitorar a própria glicemia periodicamente e fazer o registro em um dispositivo móvel, que envia estes dados para o AmbienteCicloVida.

Quadro 1 - Elementos da Realidade de Interesse.

\begin{tabular}{l|l|l|l}
\hline Evento & Natureza do Evento & $\begin{array}{l}\text { Agentes Geradores de } \\
\text { Informação }\end{array}$ & Objetos \\
\hline Aluguel de um imóvel & $\begin{array}{l}\text { Contexto de Gestão de } \\
\text { Contratos }\end{array}$ & $\begin{array}{l}\text { Locatário; Locador; } \\
\text { Imobiliária; Imóvel }\end{array}$ & - \\
\hline $\begin{array}{l}\text { Realização de exame de } \\
\text { sangue }\end{array}$ & Contexto de Saúde & $\begin{array}{l}\text { Médico; Paciente; } \\
\text { Laboratório }\end{array}$ & $\begin{array}{l}\text { Seringas, ataduras, } \\
\text { agulhas, amostra de } \\
\text { sangue ... }\end{array}$ \\
\hline $\begin{array}{l}\text { Atividade biológica medição, } \\
\text { relativa a glicemia }\end{array}$ & Contexto de Saúde & Médico; Paciente & $\begin{array}{l}\text { Aparelhos de medispositivo } \\
\text { agulha, dispón } \\
\text { móvel }\end{array}$ \\
\hline
\end{tabular}

Fonte: Própria

\subsection{Modelagem dos elementos do ambiente informacional}

Baseado nos três eventos apresentados na subseção anterior, o Quadro 2 traz as informações que devem ser geradas para os mesmos. Primeiramente tem-se o aluguel de um imóvel em que deve ser elaborado um contrato entre os agentes envolvidos (locatário; locador; e imobiliária). Também é gerado para os pacientes o documento que apresenta o resultado de um exame de sangue.

É importante observar que participam nas duas situações diversos AGIs. Por exemplo, no caso do aluguel, participam o locador, o locatário, e a imobiliária, sendo a presença desta última opcional. Neste caso, cada um dos AGIs que participa de um evento deve possuir uma conta no AmbienteCicloVida, da qual emite e recebe documentos em seus respectivos contextos.

Já a obtenção do indice de glicemia também pode ocorrer por meio de automonitoramento, em que o paciente deve, por meio do uso de algum aparelho para medir este índice, verificar e registrar o seu valor no AmbienteCicloVida.

Quadro 2 - Elementos do Ambiente Informacional

\begin{tabular}{l|l|l}
\hline Evento & Registros de Evento & $\begin{array}{l}\text { Registro de Eventos Fundamentais } \\
\text { (Agentes) }\end{array}$ \\
\hline Aluguel de um imóvel & $\begin{array}{l}\text { Contrato de Aluguel (Locador; } \\
\text { Locatário; } r \text { Intermediário- } \\
\text { imobiliária; rocador (RG/CNPJ; CPF; NOME) } \\
\text { (cláusulas; valor; prazo, etc.)) }\end{array}$ & $\begin{array}{l}\text { Locatário (RG/CNPJ; CPF; NOME) } \\
\text { Imobiliária (RG/CNPJ; CPF; NOME) }\end{array}$ \\
\hline
\end{tabular}




\begin{tabular}{l|l|l}
\hline $\begin{array}{l}\text { Realização de Exame de } \\
\text { Sangue }\end{array}$ & $\begin{array}{l}\text { Exame de Sangue (cpfpaciente; } \\
\text { crmmedico, registrolaboratório; } \\
\text { data; valor glicemia, etc.) }\end{array}$ & $\begin{array}{l}\text { Paciente (RG; CPF; NOME) } \\
\text { Médico (RG; CRM; NOME) } \\
\text { Laboratório (CNPJ; NOME) }\end{array}$ \\
\hline $\begin{array}{l}\text { Automonitoramento de } \\
\text { glicemia }\end{array}$ & $\begin{array}{l}\text { MonitoramentoGlicemia } \\
\text { (data;hora; valorglicemia) }\end{array}$ & $\begin{array}{l}\text { Paciente(RG; CPF; NOME) } \\
\text { Médico (RG; CRM; NOME) }\end{array}$ \\
\hline
\end{tabular}

Fonte: Própria

É importante ressaltar que o AmbienteCicloVida opera na internet, de modo que cada usuário possui uma conta, com acesso aos documentos e informações dos contextos apresentados.

\subsection{Modelagem dos elementos do ambiente tecnológico}

Os elementos tecnológicos que permitem o funcionamento do AmbienteCicloVida são apresentados no Quadro 3. A implementação deste ambiente foi feita em duas partes distintas:

Captura dos dados: Através das plataformas móveis mais populares atualmente (Android e iOS), os dados podem ser coletados. Informações de saúde do usuário são obtidas dos dispositivos sensoriadores e repassadas às plataformas móveis, através de conexões de redes pessoais (bluetooth);

Gerenciamento e armazenamento da informação: As informações recolhidas pelos dispositivos móveis (Android e iOS) são posteriormente encaminhadas a um servidor web e de banco de dados na nuvem. Tais servidores utilizam a linguagem PHP para interface com o usuário e a linguagem SQL para controle do sistema gerenciador de banco de dados de nome MySQL. O usuário final possui dessa forma a capacidade de aglutinar informações em um local específico para posterior resgate, o que the possibilita manter todo o seu histórico de informações.

Quadro 3 - Elementos tecnológicos

\begin{tabular}{|l|l|lll|}
\hline Evento & Hardware & Software & & \\
\hline Aluguel de imóvel & $\begin{array}{l}\text { Servidor para armazenamento } \\
\text { do AmbienteCicloVida; } \\
\text { microcomputador; dispositivo } \\
\text { móvel }\end{array}$ & $\begin{array}{l}\text { Android; iOS; Browser de } \\
\text { AmbienteCicloVida }\end{array}$ & internet; \\
\hline $\begin{array}{l}\text { Realização de exame de } \\
\text { sangue }\end{array}$ & $\begin{array}{l}\text { Servidor para armazenamento } \\
\text { do AmbienteCicloVida; } \\
\text { microcomputador; dispositivo }\end{array}$ & $\begin{array}{l}\text { Android; iOS; Browser de internet; } \\
\text { AmbienteCicloVida }\end{array}$ & \\
\hline $\begin{array}{l}\text { Autonomitoramento de } \\
\text { glicemia }\end{array}$ & $\begin{array}{l}\text { Dispositivo móvel; aparelho de } \\
\text { medição de glicemia }\end{array}$ & $\begin{array}{l}\text { Dispositivos sensoriadores; Browser de } \\
\text { internet; ambiente ciclo vida; software } \\
\text { instalado em um aplicativo móvel; }\end{array}$ \\
\hline
\end{tabular}




\section{Análise dos Resultados}

A literatura atual sobre gestão da informação e SIs assume como elemento central as organizações (LAW; NGAI, 2007; BOBSIN et al. 2010; MORESI et al. 2010; ESPANTOSO, 2012; JANUZZI et al. 2014), sendo ainda embrionários os trabalhos que focam na gestão de informações centrada nos AGIs. Com efeito, reafirma-se que apesar de todo o avanço das TICs, as principais informações de um AGI para o caso das documentais, encontram-se emitidas apenas em papel, e para o caso das não documentais, encontram-se dispersas em diferentes SIs de diferentes organizações. Por exemplo, as informações referentes a um imóvel, estão impressas na forma de papel (excrituras, registros, etc.), sob a posse do seu proprietário, bem como se encontram dispersas em SIs de diferentes tipos de organizações (prefeituras; cartórios; empresas de engenharia; imobiliárias, dentre outros agentes). Uma eventual perda de documentos do imóvel pelo proprietário o obrigaria a ter que se dirigir a estes respectivos agentes, o que lhe ocasionaria considerável tempo e custo para reaver suas cópias.

Neste artigo é proposta uma nova perspectiva para a gestão da informação, em que se defende o desenvolvimento de SIs capazes de armazenar, em ambiente de computação na nuvem, senão todas, as informações mais relevantes de um determinado AGI, de modo que elas sejam de seu livre acesso, no caso de informações de pessoas e organizações, ou pelo responsável por este, no caso de informações de objetos (automóveis, imóveis, produtos, etc.). No exemplo do parágrafo anterior, sob uma perspecgiva da GICVA o proprietário deveria ter livre acesso às informações de seu imóvel em um ambiente de computação na nuvem.

Embora tenha havido considerável levantamento bibliográfico acerca dos SIs atuais, não foi encontrada nenhuma referência paradigmática semelhante à proposta neste artigo. Todavia, verificou-se que atualmente, já existem algumas aplicações com a finalidade de armazenar as informações, principalmente no que tange o armazenamento de informações sobre pessoas em diversos aspectos de sua vida cotidiana (BRENMAN; CASPER, 2015; DOBBINS et al., 2014; GULDENPFENNING; FITZPATRICK, 2015) e iniciativas de governos no sentido de integrar dados de cidadãos, que são de interesse da administração pública (BHATIA; BHABHA, 2017; CHAUHAN; KAUSHIK, 2016; THYGESEN et al., 2011).

Há que se destacar também que o conceito de registro de informações no ciclo de vida também é mencionado em recente artigo de SALES; SAYÃO (2015) que consideram o registro das informações no ciclo de vida de atividades de pesquisa. Todavia, a proposta da GICVA aqui apresentada traz como avanços conceituais os seguintes pressupostos: o armazenamento de informações de AGIs em ambientes de computação na nuvem; o desenvolvimento de SIs para a gestão de informações oriundos de múltiplos contextos relacionados ao AGI; e uma perspectiva em que o 
AGI, sobretudo o cidadão, seja o protagonista da gestão das suas próprias informações, diminuindo a sua dependência em relação às organizações nas situações em que necessita acessá-las. Com efeito, estes vários estudos, incluindo $o$ presente artigo indicam a possibilidade de desenvolvimento de SIs, de acordo com os princípios da GICVA, em um futuro breve.

Por fim, frente ao esposto, o atual paradigma vigente de integração das informações encontra-se restrito à esfera organizacional. Contudo o desenvolvimento de aplicações GICVA apresenta a possibilidade de uma esfera de integração de informações que transcende os limites organizacionais, qual seja, a esfera universal do AGI, que ao longo de sua vida se relaciona com diferentes organizações, e segundo este paradigma deveria ter armazenadas em um único repositório, todas as suas informações, embora as mesmas possam estar replicadas em SIs de diferentes organizações.

\section{Conclusão}

Este artigo apresenta uma nova perspectiva para a ciência da informação ao propor um artefato na forma de construto à luz do método DSR. Não obstante, ele contribui para a literatura à medida que este artefato pode servir como elemento de referência para analistas e desenvolvedores de SIs, para o desenvolvimento de um novo tipo de SI, que visa atender a uma demanda social, ainda não atendida. Pois ainda nos dias atuais as informações de diversos tipos de AGIs ainda se encontram dispersas em SIs de diferentes organizações, e não são de livre acesso para o cidadão.

A implementação de SIs segundo os conceitos da GICVA propostos neste artigo permitirá a abordagem de uma série de problemas existentes hoje na sociedade, em que se destacam: as barreiras de acesso do indivíduo às informações que lhes dizem respeito; a disperção das informações relativas às entidades em diferentes SIs de diferentes organizações; o alto custo social da gestão e recuperação destas informações nestes SIs. Embora imaginar SIs capazes de gerenciar em um nível abrangente informações de diferentes tipos de AGIs, seja no momento um pensamento utópico. Sabe-se que a ciência evolui a partir de conceitos fundamentais, e mesmo de instâncias rudimentares que consistem na manifestação inicial destes conceitos. Todavia, no longo prazo, graças à evolução incremental destes conceitos e suas instâncias, aliadas a incorporação de artefatos e novas tecnologias, grandes ganhos podem ser gerados para a sociedade. Com efeito, a proposta do artefato GICVA se coloca como um passo inicial na direção do desenvolvimento de SIs com maior alcance, e que portanto permitam às pessoas e organizações um nivel de gestão da informação maior e melhor do que o vigente hoje. Embora esteja em seu estágio embrionário, o desenvolvimento de aplicações com o uso do construto proposto neste 
artigo, pode trazer grandes ganhos para a sociedade no sentido da solução destes vários problemas apontados.

Baseado no princípio epistemológico fundamental da DSR, que é gerar conhecimento científico com foco na aplicação prática e na resolução de problemas importantes para a sociedade (HEVNER et al., 2004; GREGOR; HEVNER, 2013; SORDI; AZEVEDO, 2013; SORDI et al., 2013), como continuidade desta pesquisa são sugeridas as seguintes medidas: segundo a terminologia de Peffers et al., (2017) realizar uma pesquisa para avaliação, observação e medição rigorosa da eficiência de SIs baseados na GICVA; desenvolver novos tipos de construtos para o refinamento e expansão dos conceitos sobre GICVA; abordar de forma mais profunda a gestão da informação em situações que diferentes tipos de AGIs (pessoas, objetos, organizações, etc.) participam da gênese de uma informação; analisar os impactos de SIs implementados sobre os princípios da GICVA, em aspectos como privacidade, segurança e acesso a informação; desenvolver um construto para análise do papel social/institucional que instâncias de um mesmo tipo de AGI podem assumir em um determinado contexto. Por exemplo, em uma emergência médica, o AGI do tipo pessoa pode assumir diferentes papéis, tais como médico, paciente, acompanhante, enfermeiro, etc.

Por fim, ao propor um artefato que orienta o desenvolvimento de SIs sob um novo paradigma de gestão da informação, este artigo apresenta um nova visão que mostra que as atuais TICs permitem 0 desenvolvimento de novos SIs, com maior poder de alcance do que os atuais. Estes novos SIs se implementados poderão trazer um nível de integração de dados de AGIs que ultrapassa as barreiras organizacionais. Com efeito, o desenvolvimento de SIs sob os princípios da GICVA pode trazer consideráveis avanços para as diversas organizações da sociedade e para o cidadão nos seus variados papéis sociais.

\section{Referências}

ARMBRUST, M. et al. A view of cloud computing. Communications of the ACM, v.53, n.4, p.50-58, 2010.

ASHURST, C. et al. Exploring IT-enabled innovation: a new paradigm? International Journal of Information Management, v.32, p.326-336, 2012.

BHATIA, A., BHABHA, J. India's Aadhaar scheme and the promise of inclusive social protection. Oxford Development Studies, v.45, 1, p.64-79, 2017.

BOBSIN, D.; LOBLER, M. L.; VISENTINI, M. S.; VIEIRA, K. M. O uso dos sistemas de informação e as diferenças entre os níveis hierárquicos: uma aplicação do modelo TAM-TTF. Informação \& Sociedade: Estudos, v.20, $\mathrm{n}, 3,123-134,2010$.

BRENMAN, P. F.; CASPER, G. Observing health in everyday living ODLs and the care-between-the-care. Personal and Ubiquitous Computing, v.19, n.1, p.3-8, 2015. 
CARDOSO, L. H.; PEREIRA, E. C. Teoria do caos e gestão da informação: uma integração na complexidade dos negócios e dos sistemas de informação. Transinformação, v.17, n.3, p.221-233, 2005.

CHEN, P. The entity relationship model - Toward a unified view of data. ACM Transaction on Database Systems, v.1, n.1, p.9-36, 1976.

CHANG, V. et al. The development that leads to the cloud computing business framework. International Journal of Information Management, v.33, n.3, p.524-538, 2013.

CHAUHAN,S., KAUSHIK, A. Evaluating citizen acceptance of unique identification number in India: an empirical study. Eletronic Government, an international journal, v.12, 3, 2016.

DAVENPORT, T. Ecologia da informação. São Paulo: Futura, 2000.

DING, J. H. et al. A framework of cloud-based virtual phones for secure intelligent information management. International journal of Information Management, v.34, p.329-335, 2014.

DOBBINS, C. et al. Creating human digital memories with the aid of pervasive mobile devices. Pervasive and Mobile Computing, v.12, p.160$178,2014$.

ESPANTOSO, J. J. P. E. A gestão dos espaços informacionais em ambientes de arquitetura de informação organizacional. Informação \& Sociedade: Estudos, v.22, n.3, p.33-39, 2012.

FERRO, N. et al. A formal model for digital archives. Information Process and Management, v.49, n.6, p.1206-1240, 2013.

FLORES, D.; SANTOS, H. M. Repositórios digitais confiáveis para documentos arquivísticos: ponderações sobre a preservação em longo prazo. Perspectivas em Ciência da Informação, v.20, n.2, 2015.

GREGOR, S., HEVNER, A. R. Positioning and Presenting Design Science Research for Maximum Impact. MIS Quarterly, v.37, n.2, p.337-355, 2013.

GULDENPFENNING. F.; FITZPATRICK, G. Personal digital archives on mobile phones with MEO. Personal Ubiquitous Computing, v.19, p.445$461,2015$.

HEVNER, A., MARCH, S. T., PARK, J., RAM, S. Design science in information systems research. MIS Quarterly, v.28 n.1, p.75-105, 2004.

HURSSEL, E. Idéias para uma fenomenologia pura e para uma filosofia fenomenológica. Aparecida, SP: Ideas e Letras, 2006.

JANUZZI, C. A. S. C. et al. Sistema de informação: um entendimento conceitual para a sua aplicação nas organizações empresariais. Perspectivas em Ciência da Informação, v.19, n.4, p.94-117, 2014.

HOPPEN, N.; KLEIN, A. C. Z.; RIGONI, E. H. Sociomaterial practices: challenges in developing a virtual business community platform in 
agriculture. BAR - Braziliam Administration Review, v.14, n.2, p.1-22, 2017.

KISHORE, R. et al. Enterprise integration using the agent paradigm: foundations of multi-agent-based integrative information systems. Decison Support Systems, v.42, p.48-78, 2006.

LACERDA, D. P.; DRESCH, A.; PROENÇA, A., ANTUNES JÚNIOR, J. A. V. Design Science research: método de pesquisa para a engenharia de produção. Gestão \& Produção, v.20, n.4, p.741-761, 2013.

LAUMER, S. et al. Enterprise content management. Business \& Information Systems Engineering, v.6, p.449-452, 2013.

LAW, C. H. C. et al. ERP systems adoption: An exploratory study of the organizational factors and impacts of ERP success. Information \& Management, v. 44, n.4, p. 418-432, 2007.

MAASS, W.; VARSHNEY, $U$. Design and evaluation of ubiquitous Information Systems and Use in healthcare. Decision Support Systems, v.54, p.597-609, 2012.

MORESI, E. A. D., et al. Mapeamento de informações organizacionais: um estudo na Embrapa. Transinformação, v.22, n.2, p.101-110, 2010.

NADOVEZA, D.; KIRITSIS, D. Ontology-based approach for context modeling in enterprise applications. Computers in Industry, v.65, p.12181231, 2014.

OLIVEIRA, D. P. R. Sistemas de informação gerenciais. São Paulo: Atlas, 2001.

PEFFERS, K., TUUNANEN, T., ROTHENBERGER, M. A., CHATTERJEE, S. A design science research methodology for information systems research. Journal of Management Information Systems, v.24, n.3, p.45-78, 2007.

PRADOS-SUÁREZ, B. et al. Improving eletronic health records retrieval using contexts. Expert Systems with Applications, v.39, n.10, p.85228536, 2012.

RIBEIRO, C. J. S. Serviços para gestão documental com uso de abordagem tecnológica SOA (arquitetura orientada a serviços). Informação \& Sociedade: Estudos, v.22, n.3, p.152-162,2012.

RIOS, E. R.; CORDEIRO, R. I. N. Plano de classificação de documentos arquivísticos e a teoria da classificação: uma interlocução entre domínios do conhecimento. Perspectivas em Ciência da Informação, v.15, n.2, p.123-139, 2010.

SALES, L. F.; SAYÃO, L. F. Ciberinfraestrutura de informação para pesquisa: uma proposta de arquitetura para integração de repositories e sistemas CRIS. Informação \& Sociedade: Estudos, v.25, n.3, p.163-184, 2015.

SILBERSCHATZ, A. et al. Sistema de Banco de Dados. 5. ed. Rio de Janeiro: Elsevier, 2006. 
SORDI, J. O.; AZEVEDO, M. C., MEIRELES, M. A pesquisa design Science no Brasil segundo as publicações em administração da informação. JISTEM - Journal of Information Systems and Technology Management, v.12, n.1, p.165-186, 2015.

SORDI, J. O., AZEVEDO, M. C. Proposal of a Device for the Analysis of Work Motivation Characteristics. Revista Brasileira de Gestão de Negócios, v.15, n.48, p.427-444, 2013.

THYGESEN, L. C.; DAASNES, C., THAULOW, I.; HANSEN, H. B. Introduction to Danish (nationwide) registers on health and social issues: structure, access, legislation, and archiving. Scandinavian Journal of Public Health, v.39, n.7, p.12-16, 2011.

TIGRE, P. B.; NORONHA, V. B. Do mainframe à nuvem: inovações, estrutura industrial e modelos de negócios nas tecnologias da informação e da comunicação. Revista de Administração de Empresas, v.48, n.1, p.114-127, 2013. 